Rabaska

Revue d'ethnologie de l'Amérique française

\title{
Joseph-Dominique Gauthier (1910-2006)
}

\section{Martine Roberge}

Volume 4, 2006

URI : https://id.erudit.org/iderudit/201765ar

DOI : https://doi.org/10.7202/201765ar

Aller au sommaire du numéro

Éditeur(s)

Société québécoise d'ethnologie

ISSN

1703-7433 (imprimé)

1916-7350 (numérique)

Découvrir la revue

Citer ce document

Roberge, M. (2006). Joseph-Dominique Gauthier (1910-2006). Rabaska, 4, 92-94.

https://doi.org/10.7202/201765ar

Ce document est protégé par la loi sur le droit d'auteur. L'utilisation des services d'Érudit (y compris la reproduction) est assujettie à sa politique d'utilisation que vous pouvez consulter en ligne.

https://apropos.erudit.org/fr/usagers/politique-dutilisation/
Cet article est diffusé et préservé par Érudit.

Érudit est un consortium interuniversitaire sans but lucratif composé de l’Université de Montréal, l’Université Laval et l’Université du Québec à Montréal. Il a pour mission la promotion et la valorisation de la recherche. https://www.erudit.org/fr/ 


\section{Joseph-Dominique Gauthier (1910-2006)}

Médecin et folkloriste, Joseph-Dominique Gauthier est originaire de la région de la Montérégie. Il est né le 25 février 1910 à Saint-Théodore d'Acton,

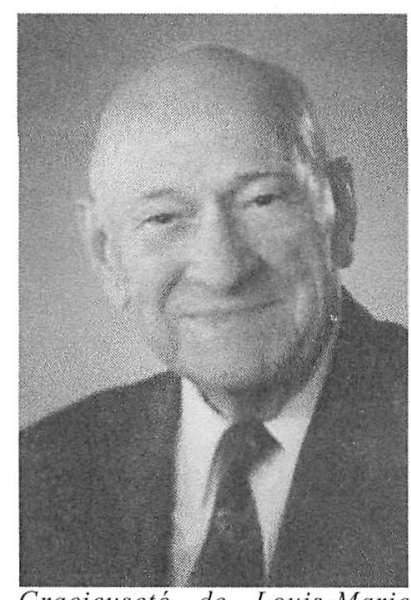

Gracieuseté de Louis-Marie Gauthier, Shippagan (communiquée par Hélène Laforte). comté de Bagot (Québec). Il est le fils de Victor et d'Odile Picard. Le 29 août 1938, il épouse Anne-Lorette LeBlanc (née le 18 juillet 1911) avec qui il fonde une famille de quatre enfants : Lise (née en 1941), Jacques (né en 1945), Louis-Marie (né en 1948) et Luce-Andrée (née en 1952). Il fait ses études au Séminaire de Notre-Dame-desCours à Papineauville puis entreprend son cours classique au Collège Saint-Joseph de Memramcook (Nouveau-Brunswick) incorporé par la suite à l'Université de Moncton d'où il détient trois diplômes : un B.A. en 1933, une maîtrise en 1941 et un doctorat en Philosophie (Ph.D.) en 1948. Cette université lui décerne aussi un doctorat honorifique. Après son baccalauréat au Collège Saint-Joseph, il s'inscrit en médecine à l'Université Laval et obtient son diplôme M.D. en 1938. Hésitant à poursuivre des études à Paris, il s'enrôle à la fin de son cours dans l'Armée impériale des Indes à titre d'officiermédecin. Avant son départ, le curé Livain Chiasson de Shippagan, qui devient par la suite un grand ami, se présente à la faculté des Sciences de l'Université Laval afin de solliciter les services d'un jeune médecin pour ses ouailles. Le curé Chiasson est alors reçu par le doyen Louis-M. Vachon qui n'hésite pas à recommander le finissant Dominique Gauthier. Ce dernier renonce alors à ses engagements d'officier et se dirige de plain-pied vers la région de Caraquet (Nouveau-Brunswick) où il commence à pratiquer la même année en 1938. Animé par le défi de pratiquer la médecine dans une région peu développée, il s'établit et travaille pendant 60 ans à Shippagan, sa terre d'adoption. À son arrivée, lui et sa femme n'ont ni eau chaude, ni électricité et les conditions de santé sont lamentables. La moitié des nouveau-nés meurent à la naissance. Sa femme, infirmière, l'assiste comme sage-femme à plusieurs reprises pour 
certains accouchements à domicile, notamment dans les îles et ce, même en hiver. De plus, elle assume la permanence du bureau à la maison lorsque le docteur Gauthier est parti en tournée de visites. Le médecin connaît bien ses patients et il développe une relation très étroite avec eux. Tout au long de sa carrière professionnelle, il pratique la médecine générale avec une spécialisation en pédiatrie. Son fils Louis-Marie, lui aussi médecin établi dans la région de Caraquet depuis 1975, raconte que son père « devait par la force des choses se faire à l'occasion arracheur de dents ou optométriste » et " que la récompense suprême était de pouvoir accompagner son père, aussi coroner, sur les lieux d'un accident " (Bulletin de l'AMLFC, 1998). La pratique du $D^{r}$ Dominique Gauthier se caractérise par le contact humain envers ses patients et son sens de l'engagement dans son milieu.

C'est par un drôle de hasard qu'il entame en parallèle une seconde carrière, celle de folkloriste. En 1950, il reçoit la visite de deux chercheurs de l'Université Laval, Luc Lacourcière et Félix-Antoine Savard. Campés à Sainte-Marie-sur-mer à l'île de Shippagan, les chercheurs ont pris froid et ils consultent Gauthier pour des maux de gorge. Référés par $\mathrm{M}^{\mathrm{gr}}$ Alphonse-Marie Parent, les deux folkloristes viennent faire la cueillette du patrimoine oral, spécialement les vieilles chansons françaises, dans le comté de Gloucester qu'ils soupçonnent d'être fertile en la matière. Dominique Gauthier est séduit par leur démarche. Il les héberge et leur indique des personnes clés à rencontrer parmi ses patients. L'aventure se poursuit pendant douze étés consécutifs et Gauthier se fait lui-même collecteur. De 1950 à 1959, il recueille quelque 300 chansons et 900 contes qu'il a légués aux Archives de folklore de l'Université Laval et qui forment l'un des plus importants fonds sur l'Acadie. La retranscription des textes de contes et de chansons est confiée à deux étudiants du programme de folklore de l'Université Laval : Gilles Vigneault et Paul de Margerie qui s'illustreront plus tard dans le domaine de la chanson. De sa collection de chansons, il en retient 70 qu'il publie en 1975 aux Presses de l'Université Laval sous le titre Chansons de Shippagan dans la collection des $"$ Archives de folklore ${ }^{1}$. Après cette publication, le folkloriste-médecin ralentit considérablement ses activités de collecte en dilettante : cette période est surtout liée à son amitié avec Luc Lacourcière qui l'a éveillé à la culture populaire acadienne.

À travers sa pratique médicale, Dominique Gauthier trouve le temps d'exercer de nombreuses activités paraprofessionnelles. Il est membre de la Commission scolaire et du centre récréatif, président fondateur de la Chambre de commerce et du Club Richelieu, secrétaire financier de la Compagnie du pont de Shippagan, membre du conseil d'administration de la Compagnie

1. Chansons de Shippagan recueillies par Dominique Gauthier. Transcription musicale de Roger Matton, [Québec], Presses de l'Université Laval, « Archives de folklore » n 16, 1975, 178 p. 
Bathurst Silver Mines Ltd et de plusieurs sociétés historiques. Il est également Commandeur de l'Ordre de la croix de Jérusalem, membre des Associés du frère André et membre de l'Ordre de Jacques-Cartier. Très engagé dans son milieu social et entièrement dévoué à son travail, il se mérite certains honneurs, dont la médaille « Pro Ecclesia et Pontifice » en 1963. Il reçoit un écusson d'honneur de l'AMLFC (Association des médecins de langue française du Canada) en 1971 et il est nommé membre émérite de la même association en 1978. Il est reçu membre de l'Ordre du Canada en 1973 pour ses travaux de folkloriste et est choisi médecin de l'année en médecine familiale par la faculté de Médecine de l'Université Laval en 1976. Il est nommé citoyen honoraire de la ville de Shippagan en 1974 et de la ville d'Acadiana en Louisiane en 1978. Il reçoit la distinction de l'Ordre du mérite de la Société médicale du Nouveau-Brunswick en 1989 et celle de Médecins de cœur et d'action de l'AMLFC en 1990. En plus de sa participation active à la vie communautaire de Shippagan, Dominique Gauthier a beaucoup voyagé et il se passionnait pour la lecture. C'est à son domicile de Shippagan au Nouveau-Brunswick qu'il s'éteint à l'âge respectable de 96 ans, le 20 mars 2006. Il laisse derrière lui un héritage précieux pour les gens de sa communauté : un sens du dévouement qu'il a su communiquer entre autres à son fils médecin LouisMarie ainsi qu'un fonds documentaire important sur la culture francophone en Amérique du Nord.

MARTINe RoBerge Université Laval, Québec

\section{Sources}

Archives de folklore et d'ethnologie de l'Université Laval, Division des Archives de l'Université Laval, "Description des fonds et des collections », Fonds Dominique Gauthier, [en ligne], http :// site.rdaq.qc.ca/archivesdefolklore, page consultée en avril 2006. Le fonds est constitué de 84 disques compacts (env. 105h). $-47 \mathrm{~cm}$ de documents textuels - dates de création du fonds $-1951-1960$.

Association des médecins de langue française du Canada, "Trois générations de médecins en Acadie », Bulletin de l'AMLFC, août 1998, [en ligne], http://www.amlfc.org/Articles/1998_08_06.html, page consultée en avril 2006.

Lalonde, Claire, « J.-Dominique Gauthier, médecin, folkloriste : découvrir Shippagan tous les jours », Journal de l'Association médicale canadienne, vol. 118, $\mathrm{n}^{\circ} 1,7$ janvier 1978, p. 88-89.

Mailhot, Carl, «Contes et chansons de l'Acadie », Perspectives, 19 décembre 1970, vol. 12, $\mathrm{n}^{\circ} 51$.

« Notice nécrologique », Le Soleil, 22 mars 2006, p. C6.

Portal, Marcel, « Dominique Gauthier, médecin », Bulletin (publié par l'Association des médecins de langue française du Canada), janv-fév. 1979, vol XIIl, $n^{\circ} 1, \mathrm{p} .27-28$

Vedettes 1958 ( $2^{\complement}$ èd.), Société nouvelle de publicité incorporée, Montréal, 1958. 